Reprod. Nutr. Dévelop., 1988, 28 (1), 209-210.

\title{
Estimation de l'eau corporelle à partir de l'espace de diffusion de l'eau deutériée chez le jeune chevreau mâle
}

\author{
Ph. SCHMIDELY, J. ROBELIN $\left({ }^{*}\right)$, P. BAS, P. MORAND-FEHR \\ Station de Nutrition et Alimentation (I.N.R.A.) de I'INA-PG \\ 16, rue Cl. Bernard, 75231 Paris Cedex 05. \\ (*) Laboratoire de la Production de Viande, \\ I.N.R.A., Theix, 63122 Ceyrat, France.
}

Summary. Empty body water, total body water at slaughter and total body water estimated in the middle of the day of injection of $D_{2} O$ (TBWM) were measured in male kids. TBWM is the compartment which is the most closely related with dilution space of $\mathrm{D}_{2} \mathrm{O}\left(\mathrm{ED}_{0}\right)\left(\mathrm{TBWM}=0.955( \pm 0.014) \mathrm{ED}_{0}, \mathrm{CVR}=3.3 \%\right)$. It provides an accurate method for body composition determination.

Chez les Bovins et les Ovins, la détermination de l'eau corporelle par la mesure de l'espace de diffusion de l'eau deutériée $\left(D_{2} O\right)$ est précise (CVR $=2 \%$ de l'eau totale) (Robelin, 1982). A notre connaissance, aucune étude n'a été effectuée chez les Caprins en croissance. Une expérimentation a donc été mise en place afin de connaître la précision de cette méthode pour estimer l'eau totale du corps chez le chevreau mâle.

Matériel et méthodes. Huit chevreaux mâles de race Saanen ou Alpine (4 implantés avec $20 \mathrm{mg}$ d'oestradiol $17 \beta+70 \mathrm{mg}$ d'acétate de trenbolone, et 4 témoins) ont exclusivement reçu du lait de remplacement jusqu'à leur abattage à 7 semaines. Deux jours avant l'abattage, une injection d'eau lourde (pureté $98 \%)$ dans la veine jugulaire a été pratiquée à l'aide d'un cathéter avant le repas $\mathrm{du}$ matin, à raison de $0,6 \mathrm{~g}$ par $\mathrm{kg}$ de poids vif. Sur 2 chevreaux, des prélèvements de sang ont été effectués $0,5,1,1,5$ et $2 \mathrm{~h}$ après l'injection afin de déterminer la durée de diffusion du traceur; sur l'ensemble des animaux, un échantillon de sang a été prélevé $2,4,6,8,24,48$ h après l'injection pour mesurer la cinétique d'élimination de $\mathrm{D}_{2} \mathrm{O}$. Une étude préliminaire a montré que l'équilibre était atteint au bout de 4 heures. La valeur de l'espace de diffusion $\mathrm{ED}_{0}$ a été calculée pour chaque animal par extrapolation au temps $t=0$ de la droite $\operatorname{Ln}$ (concentration $\left.\mathrm{D}_{2} \mathrm{O}\right)$ = a.t après équilibre. Pour réduire le biais de $E D_{0}$ dû aux variations journalières de poids vif, le poids théorique (PM) au milieu de jour de l'injection a été déterminé par régression à partir des poids vifs mesurés 15, 14, 2 et 1 jours avant l'injection.

A l'abattage, le poids (PA) et la teneur en eau du corps et des aıgesta, ainsı que les différentes quantités d'eau dans le poids vif vide (EPV), dans les digesta (ECD), dans le poids vif plein (EPA $=E P V+E C D)$ ont été mesurés par lyophylisation pendant $72 \mathrm{~h}$.

La quantité d'eau correspondant au poids vif théorique a été déterminée pour chaque chevreau par la relation : EPM $=E P A+(P M-P A) . \% \mathrm{H}_{2} \mathrm{O}$ dans les digesta, en supposant ce pourcentage constant au cours du nycthémère. 
Résultats et discussion. Les animaux implantés et témoins ne présentent pas de différences quant au pourcentage de MS dans les digesta (tabl. 1). Les équations de régression $(Y=(b \pm s b) X)$ et leur coefficient de variation résiduelle (CVR) pour chacun des compartiments $(Y)$ en fonction de $E_{0}(X)$ figurent au tableau 1. Les coefficients $b$ de chaque compartiment ne diffèrent pas significativement entre chevreaux implantés et témoins malgré une valeur systématiquement plus élevée après traitement.

TABL. 1. - Relation entre les différents compartiments aqueux corpore/s ( $\mathrm{Y}$ en $\mathrm{kg}$ ) et l'espace de diffusion $E D_{0}$ de l'eau lourde $(X$ en $\mathrm{kg}$ ).

\begin{tabular}{lcccccccccccc}
\hline & \multicolumn{3}{c}{ Eau des digesta } & \multicolumn{2}{c}{ Eau du corps vide } & \multicolumn{2}{c}{ Eau totale abattage } & \multicolumn{3}{c}{ Eau totale théorique } \\
\hline & $\begin{array}{c}\text { CVR } \\
(\%)\end{array}$ & b & sb & $\begin{array}{c}\text { CVR } \\
(\%)\end{array}$ & b & sb & $\begin{array}{c}\text { CVR } \\
(\%)\end{array}$ & b & sb & $\begin{array}{c}\text { CVR } \\
(\%)\end{array}$ \\
\hline Témoins & 91,2 & 2,3 & 0,836 & 0,029 & 6,3 & 0,913 & 0,024 & 5,3 & 0,939 & 0,021 & 4,7 \\
Implantés & 91,7 & 3,1 & 0,873 & 0,019 & 4,3 & 0,947 & 0,013 & 2,9 & 0,965 & 0,012 & 2,5 \\
Ensemble & 91,3 & 2,5 & 0,857 & 0,016 & 5,4 & 0,932 & 0,014 & 4,2 & 0,955 & 0,014 & 3,3 \\
\hline
\end{tabular}

L'espace de diffusion obtenu par extrapolation à $t=0$ sur l'ensemble des animaux est supérieur à chacun des 3 compartiments hydriques (EPV, EPA, EPM) ; il semble être un estimateur peu précis de l'eau du poids vif vide (coefficient b le plus faible et coefficient de variation résiduelle le plus élevé), ce qui s'explique par la diffusion de l'eau lourde dans le contenu digestif, puisque l'espace de diffusion n'est pas limité au corps vide. L'eau correspondant au poids vif théorique (EPM) est assez étroitement liée à $E D_{0}(b=0,955( \pm 0,014)$, CVR $=3,3 \%$ ) et le surestime d'environ $4,5 \%$, valeur légèrement plus élevée que celle obtenue par Robelin (1982) mais voisine de celle proposée par Crabtree et al. (1974), toutes deux sur Bovins. Cette surestimation, par ailleurs plus élevée chez les témoins que chez les chevreaux implantés, est un résultat assez général quelle que soit l'espèce; elle s'explique en partie par une fixation d'hydrogène marqué dans les lipides et les protéines corporels (Robelin, 1981). EPM est donc globalement mieux reliée à $E D_{0}$ dans la mesure où elle est plus représentative du volume hydrique moyen de l'animal dépendant du contenu digestif moyen de la journée.

En conclusion, l'espace de diffusion de l'eau lourde permet d'estimer in vivo avec une précision de 3,3\% l'eau corporelle totale des chevreaux de 7 semaines. Cette méthode est actuellement précisée à partir de la mesure de l'espace de diffusion de l'eau lourde chez des chevreaux âgés de 14 semaines. 